\title{
Las restricciones a la participación de los trabajadores en las organizaciones empresariales
}

\section{Joaquín Juan Albalate}

Universitat de Barcelona. Departament de Sociologia i Anàlisi de les Organitzacions

Divisió de Ciències Jurídiques, Econòmiques i Socials

Av. Diagonal, 690. 08034 Barcelona

albalate@emp.ub.es

Data de recepció: maig 2000

Data d'acceptació: novembre 2000

\section{Resumen}

La participación de los trabajadores en las organizaciones y empresas donde trabajan constituye un objetivo largamente perseguido desde que, prácticamente, se implantara la Revolución Industrial. Desde entonces, los impulsos para democratizar el trabajo no han cesado de producirse, de forma que hoy, la participación está presente - en sus diversas formas, intensidades y ámbitos - en la mayor parte de las empresas de los países occidentales. No obstante, ese proceso se ha visto condicionado y, a veces, obstaculizado por la presencia de ciertos idearios, así como por un conjunto amplio de factores, de cuya conjunción se ha derivado que la participación se haya estabilizado como un fenómeno social positivo y aceptado por los diversos interlocutores o, por el contrario, no se haya estabilizado como tal o, si lo ha hecho, haya sido en términos de conflictividad.

Palabras clave: participación, trabajadores, condicionantes, ideología.

\section{Resum}

La participació dels treballadors al si de les organitzacions i empreses on treballen constitueix un objectiu llargament perseguit des que, pràcticament, s'implantés la Revolució Industrial. Des d'aleshores, els impulsos per democratitzar el treball no han cessat d'esdevenir-se, de tal manera que avui, la participació és present —en les seves diverses formes, intensitats i àmbits - a la major part de les empreses dels països occidentals. No obstant això, aquest procés s'ha vist condicionat $\mathrm{i}$, a cops, obstaculitzat per la presència de certs idearis, alhora que per un conjunt ampli de factors, de la conjunció dels quals s'ha derivat que la participació s'hagi estabilitzat com a fenomen social positiu i acceptat pels diversos interlocutors o, per contra, no s'hagi estabilitzat com a tal o, si ho ha fet, s'hagi produït en termes de conflictivitat.

Paraules clau: participació, treballadors, condicionants, ideologia.

\section{Abstract. Restrictions to the participation of workers in the business organizations}

The participation of workers in the organisations and enterprises, for which they work, constitutes along time seeked objective, practically since the Industrial Revolution was implanted. Since then, the impulses to democratise have never stopped taking place, so that today, participation is present - in its different ways, intensities and areas - in most 
of the enterprises of the occidental countries. However, such process has been conditioned and, sometimes, prevented by the presence of certain ideologies and as well as by a wide set of factors, of which conjunction, it is derived that the participation it has stabilished as a positive social phenomenon and accepted by the different interlocutors or, on the contrary, has not done it or if so, has been in terms of conflictivity.

\section{Sumario}

Introducción 4. Objetivos de las organizaciones

1. Las corrientes teóricas de la participación

2. Concepto y tipos de participación en las organizaciones

3. La legislación participativa en España y potencial democrático

5. La capacidad de los trabajadores para participar

6. Los condicionantes de la participación

7. Algunas conclusiones

Bibliografía

\section{Introducción}

La idea que subyace a la necesidad de que los trabajadores participen en las decisiones que se toman en las organizaciones y empresas de las que forman parte, obedece a un razonamiento, en principio, lógico y comprensible: si la democracia, como sistema político de gobierno, está extendida en las sociedades modernas como forma social seleccionada, política y culturalmente, para elegir a los representantes de los ciudadanos que han de decidir sobre los destinos de esa sociedad y de ellos mismos, por qué ese mismo sistema de toma de decisiones conjunta del que se han dotado esas sociedades para autogobernarse, no traspasa la frontera de la empresa y se traslada también a su funcionamiento interno, más cuando ésta es parte integrante de esa misma sociedad.

De esa forma, mientras en la sociedad civil de estas sociedades predomina un sistema de valores democráticos, en el ámbito económico y laboral siguen rigiendo otro tipo de valores ajenos al comportamiento democrático, con lo que muchas personas viven una experiencia social «escindida», es decir, mientras «[...] fuera del trabajo [...] los individuos son tratados como ciudadanos; sin embargo, de puertas adentro del trabajo, son tratados como "súbditos" y obligados a comportarse como tales [...]» (Tezanos, 1987: 21).

Hoy está ampliamente reconocido que la participación de los trabajadores en los procesos de toma de decisiones y en un mayor control por parte de éstos mismos sobre su propio trabajo, son soluciones necesarias, aunque no suficientes, para intentar resolver los problemas — algunos de ellos enormemente complejos - que comporta el trabajo en nuestra era. Sin embargo, como luego se podrá comprobar, la plasmación a la realidad de la idea participativa ha sido más bien endeble y limitada. En la mayor parte de los casos en que se ha intentado llevar a cabo, no ha supuesto la participación activa de los trabajadores 
(Castillo, 1987: 21), al menos en el sentido más originario del concepto de democracia o, en otras palabras, de participación directa y consciente y no de mera delegación pasiva.

Si la empresa no evoluciona a la par que lo hace su entorno, puede llegar a convertirse en un ente crecientemente inadaptado a su ambiente con resultados negativos en lo económico, así como incongruentes en lo social, sobre todo cuando en las teorías más avanzadas sobre la organización social se postula que el desarrollo de la empresa pasa por el desarrollo de las personas que trabajan en la misma (Parra, 1993: 165).

Las ideas que hoy existen sobre la participación deben verse también como un ulterior contrapunto a la tendencia histórica hacia la alienación en el trabajo de los trabajadores, ya indicada por A. de Tocqueville en su obra La democracia en América, publicada en 1835, así como por otros autores contemporáneos a éste, como Mill, Marx o Durkheim. Sin embargo, se ha de reconocer que los esfuerzos por combatir la alienación y la enajenación de los trabajadores en las fábricas — ampliando su capacidad para decidir sobre su propio trabajo-, no empezaron a fructificar con fuerza hasta pasada la Segunda Guerra Mundial, a partir de la cual cristalizaron determinadas experiencias, en paralelo a ciertas posiciones teóricas críticas, ambas surgidas en el marco del nuevo sistema de relaciones laborales posbélico.

La implantación y desarrollo del Estado de bienestar en las sociedades democráticas, tras aquella contienda, favoreció una amplia difusión de las ideas y de los valores democráticos, con lo que se consiguió generar un clima de opinión pública propenso a exigir que, cada vez más, todas las esferas de la vida social tendieran a organizarse de acuerdo con los procedimientos democráticos.

Desde entonces y, especialmente, desde los años sesenta, el auge de las propuestas para reducir la alienación en el trabajo y, más allá de ese aspecto inicial, del impulso para lograr un incremento del control democrático de la actividad global de las organizaciones empresariales, no ha dejado de cesar por todos los países occidentales, en consonancia con los propios progresos alcanzados en la democratización política y social, que han hecho menos tolerable la persistencia de estructuras autoritarias en el plano económico (Tezanos, 1987: 23), lo que ha dado lugar a la emergencia de una nueva etapa en la evolución de la democracia industrial hacia formas de participación más amplias y directas, conforme la nueva sociedad tecnológica ha ido abriendo nuevas oportunidades para la participación.

\section{Las corrientes teóricas de la participación}

Los orígenes de los primeros estudios de los efectos de la participación en el contexto del mundo del trabajo, se remontan a los experimentos de Hawthorn llevados a cabo por Elton Mayo durante los años veinte y treinta de este siglo, como miembro destacado de la Escuela de Relaciones Humanas, en los que se pudo comprobar la asociación entre la elevación de la motivación de los trabajadores y el aumento de la productividad de la empresa, como consecuencia 
del reconocimiento explícito de ésta del trabajo de sus miembros, invocando de esta manera la importancia de su presencia y participación en la consecución de los objetivos perseguidos por la empresa. No obstante, hay que señalar que, ya Taylor, no sólo no descartó la participación de los trabajadores, sino que la vio imprescindible en su filosofía sobre la dirección científica de la organización del trabajo. Sin embargo, la puesta en práctica del taylorismo por parte de sus discípulos y numerosos empresarios que siguieron sus principios, casi nunca tuvo en cuenta este aspecto, por lo que la participación de los trabajadores no se activó, y ocurrió lo mismo con otros preceptos importantes de esta corrientes organizativa, como son la necesidad de formación o de un salario suficiente para los trabajadores.

Por todo ello, el significado que adquirirá la participación para la Escuela de las Relaciones Humanas será de simple instrumento para lograr la cooperación de los trabajadores y obtener de ellos el acatamiento a las decisiones directivas, de una forma menos costosa que el recurso a la coacción, utilizada a menudo por el taylorismo. Por tanto, a pesar de que supondrá un salto cualitativo en la «humanización» del trato a los trabajadores respecto al taylorismo clásico, la participación sólo será un accesorio potencial a utilizar cuando aumenten las tensiones y los conflictos, para restaurar el estado de ánimo del individuo y mejorar el ambiente de trabajo, sin que ello implique la modificación de la raíz de los problemas causantes de estas tensiones, ni el cambio de las relaciones de poder entre los diversos grupos profesionales, en favor de una mayor participación de todos los miembros en la toma de decisiones de la empresa.

La aparición del modelo de recursos humanos durante los años sesenta en Estados Unidos, en reacción a la escuela anterior, significó el punto de partida para un cambio substancial de la perspectiva de la participación. Se parte de la idea de que todos los individuos que integran la organización poseen, no sólo habilidades técnicas y físicas, sino también una serie de capacidades personales, fruto de una mayor educación y del avance social — como las relativas a la creatividad, a la de responsabilidad, de autonomía, de autocontrol, etc.que habrán de condicionar, necesariamente, la que habrá de ser la nueva tarea directiva, centrada, desde ahora, en la creación del ambiente adecuado para que las potencialidades y capacidades de todos los recursos humanos de la organización se puedan emplear, de forma que el trabajo resulte satisfactorio para el conjunto de los miembros de ésta.

Desde esta nueva perspectiva, el aumento de la satisfacción de los trabajadores no será ya la causa de un mejor desempeño, sino de las contribuciones que éstos realizarán en la toma de decisiones para el progreso de la organización (Quintanilla, 1987: 27). El directivo, por su parte, no sólo debe permitir la participación en las decisiones de rutina, sino que ha de estimularla de forma que «[...] cuanto más importante sea la decisión, mayor debe ser su obligación para alentar a los subordinados para que propongan nuevas ideas y sugerencias [...]» (Quintanilla, 1987: 28).

Con posterioridad, en los años setenta y ochenta, dentro de la moderna 
teoría organizacional más reciente, surgirá un gran número de modelos y técnicas directivas, pertenecientes todos ellos, en mayor o menor grado, al denominado "modelo participativo" (Tezanos, 1987: 41), los cuales, a pesar de poner el acento en aspectos específicos distintos sobre la concepción y puesta en práctica de la participación, van a confluir en utilizar a la participación como el elemento organizacional básico, no sólo por su carácter instrumental para que los individuos y grupos de trabajo propongan cambios y mejoras tecnológicas y organizativas, sino para que sean ellos mismos los que tomen las decisiones más adecuadas en las empresas.

Es el caso, entre otras, de las técnicas para la «humanización del trabajo» y el desarrollo de la «calidad de la vida laboral» en Europa o la implantación de la «teoría Z" de Ouchi en Estados Unidos, por no hablar de los "círculos de calidad» japoneses - a pesar de la visión paternalista y jerarquizada de la participación en la que se inscriben-, en todas las cuales la participación de los trabajadores se convierte en el eje central sobre el que gira el resto de sus actuaciones.

Sin duda, uno de los modelos más característicos de participación, coincidente en bastantes aspectos con las aspiraciones de la corriente de Recursos Humanos, y que más ha impactado en la literatura relacionada con el tema por la profundidad de los logros obtenidos, ha sido el de la «democracia industrial» en Suecia. La larga tradición democrática de este país en el ámbito político, se concretó, ya desde los primeros acuerdos firmados en 1938 y de los sucesivos pactos a lo largo de todo este siglo entre trabajadores y empresarios, en un compromiso para potenciar la participación de los trabajadores también en el ámbito laboral, con unos niveles de profundidad comparados con los conseguidos en los otros ámbitos sociales, convirtiéndose en una experiencia de referencia para la participación organizacional para el resto de países.

Los objetivos finales del modelo de "democracia industrial», aun coincidiendo en última instancia con los que, en general, persiguen los otros modelos anteriores, es decir, lograr las condiciones de trabajo necesarias para motivar la implicación del trabajador en la empresa; se centran en la búsqueda de la satisfacción y el enriquecimiento pleno en el trabajo, no sólo desde una óptica horizontal, sino también desde la vertiente vertical del poder, profundizando en las prácticas democráticas en todas las fases del proceso de toma de decisiones, considerando el resultado de la producción de bienes como un resultado colectivo y no individual de cada trabajador.

Finalmente, desde una visión más conceptual que experimental, el tema de la participación (Finkel, 1994: 352) se ha situado recientemente entre dos polos de reflexión extremos: por un lado, se ha situado Harry Braverman, quien defendiendo la pervivencia de un taylorismo/fordismo «renovado» en el funcionamiento organizativo de las empresas, presupone que la implicación o participación de los trabajadores en éstas, no sólo no modifica sustantivamente las tendencias a su degradación y alienación, sino que constituye una estrategia "cosmética» para afrontar con mayor éxito las exigencias de un mayor control que el mercado actual reclama del empresario, ante las graves conse- 
cuencias que un hipotético conflicto podría tener para los objetivos empresariales.

La participación "cosmética» es fundamental para la adhesión ideológica de los trabajadores, de forma que éstos hagan suyos los objetivos de la empresa y, por tanto, descarta de entrada la coerción como medio de control, dados los escasos resultados obtenidos por este medio en el pasado. De hecho, las tesis manifestadas por Braverman han sido, en mayor o menor grado, compartidas también por otros autores críticos como Burawoy, Polanyi, Edwards, etc. y, especialmente, por una parte del mundo sindical cuando, a la vista del alcance real de la participación en numerosas organizaciones y de las estrategias iniciadas por las propias empresas en esa materia, han interpretado el fenómeno más en términos de manipulación, que como una verdadera intención de la dirección de hacer partícipes a los trabajadores en las decisiones realmente importantes, y no sólo en las meras decisiones de carácter secundario, en última instancia, tomadas también bajo la hegemonía empresarial.

Por el otro polo de reflexión, desde una visión claramente "optimista», tanto para Piore y Sabel, cuando hablan de un giro histórico hacia la «especialización flexible», como para Kern y Schuman, cuando anuncian el fin de la división del trabajo, dan por agotado el fordismo y afirman que las nuevas formas de organización del trabajo que se están introduciendo en las organizaciones productivas, van a favorecer un trabajo más gratificante y liberador, y que, por tanto, no sólo se va a facilitar, sino que se va a exigir la participación y el compromiso de los trabajadores, aunque sin especificar quien, sobre qué ni cómo llegará a hacerse.

En definitiva, la participación como concepto teórico y como práctica real ha seguido una evolución sinuosa, pero, a pesar de todo, ascendente, sujeta a la inevitable influencia que, en cada momento y lugar, han tenido determinados factores internos y externos a las organizaciones, y que han conformado una estructuración concreta de la participación en cada organización.

\section{Concepto y tipos de participación en las organizaciones}

Desde el punto de vista etimológico, el término participar significa «acción de tomar parte en algo». No resulta tan fácil, sin embargo, definir qué se entiende por la idea de "participar en una organización o empresa» o, menos aún, por «democratizar el trabajo», u otras expresiones más o menos equivalentes, porque esa acepción adquiere diferentes significados y connotaciones en función de los objetivos e intereses que cada individuo o agente interventor persigue con la participación, por lo que la ambigüedad suele estar presente en la mayor parte de las definiciones y clasificaciones que se realizan sobre este concepto.

El concepto de participación encierra, inevitablemente, un aspecto político, al no poder disociarse de cuestiones de autoridad, legitimidad, poder y control, por lo que abarca actitudes diversas y conflictivas cuando se intenta llevar a la práctica, en tanto que incide en las concepciones sociales y políticas que los distintos interlocutores poseen sobre, por ejemplo, el cómo debería de 
regularse la participación en la propiedad de la empresa y/o cómo habría que distribuir las atribuciones de poder en la gestión de ésta.

Por tanto, las dificultades para definir lo que significa la democratización del trabajo, no sólo provienen de su carácter aún de ideal, dado que no se ha concretado lo suficiente todavía como para poder determinarla con cierta precisión, sino porque las aproximaciones que se ha hecho se han desarrollado bajo modelos y dimensiones de alcance muy distintos.

La democratización de las empresas admite distintos tipos de participación de los trabajadores en las organizaciones en función de un conjunto de variables sobre las que cada autor pone su acento particular. Así, mientras para Quintanilla, la participación se lleva a cabo mediante el establecimiento de metas, la toma de decisiones en la solución de problemas o en el cambio organizativo (Quintanilla, 1988: 113), en cambio para Tezanos la democratización del trabajo constituye un proceso complejo que puede ser objeto de múltiples concreciones, según es el grado de acceso que tienen los trabajadores para participar en las empresas. Así, este autor habla de dos dimensiones básicas implícitas en el concepto de democratización del trabajo en la empresa: la que se refiere al control de la gestión y la que se relaciona con la propiedad. A partir de estas dos grandes esferas susceptibles de participación, se puede clasificar a las organizaciones empresariales en cuatro tipos (Tezanos, 1987: 27): «tradicionales», "capitalistas modernas», "colectivizadas» y «cooperativas y autogestionadas», en función de la intensidad, de menos a más, con la que los trabajadores de cada uno de esos tipos de organización pueden alcanzar un mayor o menor grado de poder en el control y la propiedad de las empresas.

Desde una óptica parecida a la anterior, Finkel desdobla la participación sobre el control de la gestión de la empresa en dos subáreas: la de los resultados y la de la organización interna del trabajo (Finkel, 1995: 352), afirmando que, a pesar de ciertas tendencias contradictorias sobre el alcance real de la participación de los trabajadores en las empresas, puede afectar a tres grandes áreas:

a) La que se refiere a la participación en la propiedad del capital de la empresa en forma de acciones, con lo que los trabajadores contraen el derecho a codecidir en la toma de decisiones del consejo de administración de aquélla.

b) La que se refiere a la participación en los resultados de la empresa, cuya manifestación más generalizada se concreta en la obtención de pagas extraordinarias por beneficios, productividad $\mathrm{u}$ otro tipo de remuneraciones salariales o en especie.

c) La que se refiere a la participación en la gestión, es decir, en cómo organizar la actividad ordinaria de la empresa, y que abarca desde el mismo momento en que se deciden los objetivos a alcanzar a los modos de organizar y distribuir las tareas y los puestos de trabajo. Este tipo de participación es el que ha generado más literatura, ya que afecta al núcleo de las 
prerrogativas que poseen los empresarios sobre el tipo de las relaciones que se establecen en la producción.

Por su parte, Moro propone observar la participación, simplemente, a partir del grado de implicación que se deriva del acuerdo entre las partes (Moro, 1977: 101). Así, según este autor, mientras la "codeliberación» implica una mera aportación de «visiones» de ambas partes, sin que ello comprometa a la dirección a tomar en consideración todo lo aportado por los trabajadores; la «codecisión» o «codeterminación» consiste en un avance en la capacidad de los trabajadores para acordar, con carácter vinculante y conjuntamente con la dirección, las decisiones que afectan a la empresa. Finalmente, la «cogestión» o "codeterminación» se situaría en un estadio superior, en el que existe una equidad de poder de partida para gestionar y decidir sobre la totalidad de los medios y los fines de la empresa, por lo que la iniciativa puede proceder de ambas partes, sin que una pueda imponer sus criterios a la otra.

También puede estudiarse la participación desde la fuente que da pie a su origen y legitimidad (Divar, 1990: 91). Así, la participación puede provenir del «otorgamiento», cuando ésta proviene de la cesión que la empresa hace, unilateralmente, a los trabajadores. Puede proceder del "convenio", como fruto del acuerdo pactado libremente entre ambas partes, tanto a nivel de empresa, como colectivamente, entre representantes sectoriales o nacionales de esas partes. Finalmente, la participación puede establecerse a través del ordenamiento jurídico por el legislador, con lo que adquiere carácter de obligado cumplimiento en todo el territorio donde rige la ley.

Por último, tanto Hermel (Hermel, 1990: 119) como Giménez (Gimenez, 1995: 141), proponen una visión de la participación más completa y detallada, a partir de combinar la naturaleza de la participación con la intensidad de ésta. Así, según estos autores, la participación de los trabajadores puede darse en:

a) Los medios o recursos, mediante aportaciones financieras que, como ya se ha visto anteriormente, permiten, normalmente, el acceso al accionariado de la empresa y, con ello, la obtención de un cierto control sobre las grandes directrices de ésta, sin que ello dé derecho a la participación en las decisiones sobre su organización y funcionamiento.

b) El proceso o funcionamiento, como resultado implícito de la propia actividad laboral en la ejecución del trabajo en la empresa, aunque no por ello eso ha de significar, necesariamente, participar en la concepción o diseño de ese trabajo, que es la forma más completa de participar en el proceso que tiene el trabajador (Hermel, 1990: 125), porque le permite definir, concertadamente con el resto de trabajadores y responsables, los objetivos de su trabajo y organizar la estrategia y los medios más adecuados para alcanzarlos.

c) Los resultados, o todo tipo de recompensas materiales o inmateriales que los trabajadores pueden obtener, tanto por su buen rendimiento individual 
como por los beneficios alcanzados por la empresa por el buen funcionamiento del proceso de trabajo global. Sin embargo, este tipo de prácticas, altamente extendidas, han sido objeto de crítica por su cuestionable carácter de verdadera participación, en tanto que suelen estar vinculadas a una participación limitada a la mera ejecución del trabajo, además de que pueden estar sujetas a criterios que pueden dar lugar a la "antiparticipación» de los sujetos (Giménez, 1995: 142).

Estos tres grandes ámbitos de participación en la empresa están íntimamente relacionados, a su vez, con otras tantas variables que permiten concretar la amplitud y la intensidad de esa participación:

a) El grado de participación, referido al tiempo de la acción participativa (coyuntural o permanente) y a la extensión en el espacio de ésta a un sólo ámbito de los tres anteriormente citados (parcial) o, por el contrario, a todos ellos (global).

b) Los actores afectados, referido a la amplitud de los grupos sociales que quedan involucrados en las prácticas participativas, en el sentido de si sólo afectan a unos (usualmente, directivos o técnicos) o, contrariamente, implica a todos los niveles de la organización que voluntariamente lo deseen. Más aún, desde el punto de vista de la amplitud, la participación ha de considerar también a los agentes sociales (patronales, sindicatos y la Administración), en tanto que actores, directa o indirectamente, implicados en la participación (Giménez, 1995: 144), por la importancia que sus decisiones (acuerdos marco generales, convenios colectivos, normativas al respecto, etc.), pueden tener — particularmente las de los sindicatos- para que la dinámica de la participación en las empresas sea aceptada y profundizada con el menor grado de conflictividad posible.

c) La forma de participación, que hace referencia a los niveles de intensidad, en orden creciente, con la que los trabajadores pueden participar en las decisiones de la empresa. Así, de forma equivalente a lo propuesto anteriormente por Moro, se puede hablar de participación en grado de «información», "consulta», "codecisión» o «autogestión», según sea el poder que tienen los trabajadores para ser sólo informados por la dirección, ser consultados pero no necesariamente obedecidos, tener que contar, obligatoriamente, con su criterio para tomar una decisión o, simplemente, tomar las decisiones por ellos mismos en tanto que socios de la empresa (caso de las cooperativas de trabajo asociado o las sociedades anónimas o limitadas laborales, en el caso de España). También puede considerarse la forma de participación, observando si es "directa», es decir, cuando un individuo o grupo tiene reconocido el poder para ejercer influencia en su entorno inmediato tomando las decisiones necesarias de forma autónoma o, por el contrario, si es «indirecta», es decir, realizada a través de intermediarios, como por ejemplo la que se lleva a cabo a través de entes como los comités de empresa (Hermel, 1990: 135). 


\section{La legislación participativa en España}

La consolidación, con carácter de «derecho», de la participación de los trabajadores en las empresas, tal como ha sucedido con la mayor parte de los derechos que han adquirido carácter de ley, ha sido el resultado de un proceso de adecuación de las normas jurídicas a las circunstancias sociales e históricas en las que una comunidad determinada - en este caso, las sociedades democráticas occidentales - lo ha exigido como valor de «justicia social» (Divar, 1990: 25), en la medida que esa comunidad ha entendido que la aplicación de tal derecho puede ser evitador, protector o/y regulador de potenciales conflictos derivados de la existencia de intereses enfrentados.

Según Divar, «[...] las concepciones y doctrinas sobre las aspiraciones justas [...] cambian en el transcurso de los tiempos dentro de los mismos pueblos y los mismos grupos [...]" (Divar, 1990: 26), por lo que, si la comunidad de hoy ha entendido que la participación en el trabajo es un bien social, un valor de justicia social, es perfectamente reivindicable como derecho positivo, más si se tiene presente que las empresas que actúan en el seno de las sociedades democráticas están afectas al interés social general y, por eso mismo, es posible imponer los controles y beneficios sociales (de participación laboral o de controles medioambientales, por sólo citar algunos), sin que por ello se justifiquen los intervencionismos atentatorios a los derechos de particulares, ni se vulnere el derecho a la libre empresa (Divar, 1990: 88).

La introducción de la participación de los trabajadores en la empresa en las legislaciones europeas se inicia a raíz de las exigencias impuestas por la reconstrucción económica de los territorios devastados, a consecuencia de las secuelas derivadas de la Segunda Guerra Mundial. En un principio, la participación se circunscribió a cuestiones relacionadas con el bienestar social y personal de los trabajadores, en consonancia con el desarrollo del Estado de bienestar que por entonces iniciaba su despliegue en la mayor parte de los países occidentales para, a partir de la década de los setenta, dar un salto cualitativo y extender la participación hacia el ámbito financiero de la empresa, tanto en relación con la política de inversiones como en lo referente a la gestión propiamente dicha.

El tratamiento jurídico de la participación de los trabajadores en la empresa en los países europeos no es unívoco, pudiéndose distinguir dos «modelos» diferenciados: el denominado de "co-gestión», en el que los trabajadores quedan implicados en los órganos internos de gestión o vigilancia de la empresa que, con variantes más o menos acusadas, se desarrolla en Alemania, Austria, Dinamarca, Noruega o Suecia; y otro modelo basado en el control de los trabajadores, ejercido desde órganos externos a los de la empresa — fundamentalmente, los comités de empresa-, articulado en base a la negociación confrontación, propio de países como Italia, Francia y, en cierta medida, el resto de países del sur de Europa.

La constatación de esta diversidad legislativa sobre el derecho a la participación de los trabajadores europeos ya fue objeto de preocupación de las auto- 
ridades comunitarias cuando en 1975 ya se intentó, aunque sin éxito desde entonces, armonizar ese campo. Así, en el Libro verde sobre participación de los trabajadores en las estructuras de las empresas de ese año, se destacaba la exigencia de que convergieran las diversas legislaciones europeas en materia de participación.

Desde el marco comunitario, con base en el artículo 100 del Tratado de la Comunidad Económica Europea de 1956, la Comisión de las Comunidades ha realizado diversos intentos de armonizar esta materia, mediante ciertas directivas como la de Reforma de las Sociedades Mercantiles de 1976, que autoriza a las sociedades a dar facilidades financieras al personal para la adquisición de acciones de la propia empresa (Giménez, 1995: 256), u otras directivas como la 75/129/CEE, sobre despidos colectivos, la 77/187/CEE, sobre derechos de los trabajadores en los casos de transmisión de empresas, o las tercera y quinta directivas - 78/855/CEE y 82/891/CEE, respectivamente- sobre fusión y escisión de sociedades.

Otros artículos como el 129.2 o el 9.2 de ese tratado, insisten en que «los poderes públicos han de promover con eficacia las condiciones para que se implanten las diversas formas de participación en la empresa» y "remover los obstáculos que dificulten o impidan su plena adopción». Sin embargo, y a pesar de que todo ese conjunto legislativo sólo hacía referencia a derechos de información y consulta a los trabajadores, los resultados que se han cosechado durante todos estos años han sido más bien escasos.

Por lo que se refiere a España, el derecho a la participación de los trabajadores está reconocido por la Constitución española en su artículo 129, apartados 1 y 2 , donde se establece que «[...] Los poderes públicos promoverán eficazmente las diversas formas de participación en la empresa y fomentarán, mediante una legislación adecuada, las sociedades cooperativas. También establecerán los medios que faciliten el acceso de los trabajadores a la propiedad de los medios de producción [...]». Sin embargo, a pesar de ese mandato constitucional, no se ha producido un desarrollo legislativo posterior suficientemente abundante ni adecuado, por lo que, hasta la actualidad, no existe una relación entre la regulación legislativa y la práctica participativa que se lleva a cabo en España.

En el Acuerdo Marco Interconfederal (AMI) de 1980, firmado por la UGT y la patronal CEOE, se plasmaron una serie de contenidos en materia de participación en la empresa que más tarde serían recogidos por el Estatuto de los Trabajadores, en su artículo 64, aprobado en 1983, donde se establecieron las funciones de los delegados sindicales y de los comités de empresa, así como los derechos en materia de negociación colectiva y la firma de convenios colectivos.

La Ley Orgánica de Libertad Sindical de 1985, además de institucionalizar las secciones sindicales en las empresas y dar carácter de ley al derecho a la huelga, facultará a los sindicatos más representativos para participar como interlocutores institucionales en ciertos organismos de las administraciones públicas, así como en los sistemas no jurisdiccionales de solución de los conflictos. 
En definitiva, la legislación española existente es, prácticamente, de «mínimos», sin que exista un modelo legislado de participación de los trabajadores en las empresas, por lo que, frente a ese vacío jurídico de facto, los trabajadores españoles han ido elaborando toda una doctrina de la participación en su quehacer cotidiano, basada, sobre todo, en la experiencia laboral y sindical, con lo cual casi todos los avances efectivos que se han conseguido en la participación en España, se han plasmado primero en los convenios colectivos o, en todo caso, en acuerdos institucionales generales entre los agentes sociales, limitándose las leyes, en el mejor de los casos (Saracíbar, 1987: 203), a consagrar posteriormente esos avances.

La precaria legislación sobre la participación de los trabajadores en España, ha visto su confirmación, en los tiempos más recientes, por la posición contraria manifestada por la representación gubernamental española a la recuperación de ese tema por parte de la Comisión Europea, a partir del denominado "Grupo de Davignon» y de los dos informes «Pepper» de 1991 y 1996 para el fomento de la participación de los trabajadores en los resultados de las empresas que, durante los últimos años noventa, intentan alcanzar un consenso en la regulación de un estatuto de sociedad mercantil europea, proyecto que resta bloqueado desde hace más de veinticinco años. En este sentido, el acuerdo sobre la prevalencia de la legislación europea más favorable para los intereses de los trabajadores, en cuanto a mantener los derechos de éstos en el control y la vigilancia de sus intereses ante los efectos de la fusión de sociedades de distintos países de la Unión Europea para la creación de una nueva sociedad, ha quedado bloqueado por la oposición de la delegación española, convirtiéndose en el único país de los quince que frena el intento de homologar jurídicamente un espacio común europeo para la participación de los trabajadores.

\section{Objetivos de las organizaciones y potencial democrático}

Tanto factores estructurales, como culturales y motivacionales hacen que la participación colectiva en el gobierno de la organización sea más o menos dificultosa de implantar. Así, por ejemplo, haciendo referencia a la clasificación que Blau y Scott realizaron durante los años sesenta sobre las características de las organizaciones formales (Castillo, 1987: 334), estos autores distinguían cuatro tipos de personas que forman parte de las organizaciones: a) los miembros ordinarios o participación corriente; $b$ ) los propietarios o directores de la organización; c) los clientes y, en general, el público que tiene contacto directo y regular con la organización, y d) el resto de personas de la sociedad en la que la empresa opera.

Desde esa clasificación de las personas, a su vez, se ordenaba a las organizaciones de acuerdo con el criterio cui bono, o quién se beneficia de las actividades de la organización. Así, en correspondencia con la anterior taxonomía, el primer tipo de personas quedan asociadas a organizaciones de índole mutuobenéficas, cuyos principales beneficiarios son los miembros ordinarios (desde sindicatos hasta colegios profesionales o partidos políticos). El segundo tipo se 
refiere a empresas mercantiles cuyos beneficiarios principales son los propietarios y los directivos. Los que forman parte del tercer tipo (hospitales, universidades, centros recreativos, etc.) quedan ligados a organizaciones de servicios cuyos principales beneficiarios lo constituyen los clientes, y, finalmente, el cuarto tipo está ligado a organizaciones públicas (Ejército, servicios municipales de incendios, etc.), cuyo principal beneficiario es el público en general.

En ese sentido, si esa clasificación se lleva al campo de la participación, el objetivo y, a la vez, problema básico que tienen las organizaciones mutuobenéficas es el de procurar la participación de los asociados en el gobierno de éstas, en cambio, el objetivo de las empresas mercantiles es el de lograr la eficiencia máxima, mientras que el de las organizaciones de servicios es el de atender lo mejor posible a la clientela y, finalmente, el de las organizaciones públicas, el de velar por el establecimiento de procedimientos democráticos que permitan a la sociedad vigilar su funcionamiento.

En cualquiera de los cuatro casos descritos, cuando se produce un cambio en la organización que altera el estatus del principal beneficiario, se producen importantes transformaciones en la naturaleza de la organización (pasando de un tipo a otro, por ejemplo, por substitución del principal beneficiario). Si estas ideas se aplican rigurosamente a la democracia industrial en las empresas, puede resultar que ésta podría suponer un intento de convertir una organización mercantil en una asociación mutuobenéfica, con lo que el principal objetivo de la organización dejaría de ser la búsqueda del máximo rendimiento económico, por medio del estricto funcionamiento de la cadena de mando, para ser el de garantizar su propia democracia interna. Por tanto, si lo que se desea es la eficacia operativa de la organización, no parece que la participación de los trabajadores se pudiera convertir en el objetivo fundamental de este tipo de organizaciones.

Sin embargo, los resultados de la mayor parte de los numerosos experimentos que se han llevado a cabo durante las últimas décadas en las organizaciones industriales (Castillo, 1987: 336), han mostrado que los grupos participativos se muestran, casi siempre, más satisfechos con el trabajo que realizan y más identificados con los objetivos de las organizaciones para las que trabajan, que los grupos no participativos, por lo que, a pesar de esa aparente contradicción apuntada antes, entre búsqueda de beneficios y participación de los trabajadores, nada hace pensar que esos objetivos sean, necesariamente, incompatibles, por lo que se deduce que la participación no admite clasificaciones ni correlaciones inflexibles, en tanto que fenómeno adaptable a una gran diversidad de situaciones organizativas, en función de otros muchos factores que intervienen para que, finalmente, la participación sea o no una realidad.

\section{La capacidad de los trabajadores para participar}

En general, la mayor parte de los estudios han constatado que la participación de los individuos en las organizaciones productivas ha tenido resultados positivos (Fundación...,1988: 51). Así, a nivel individual, la participación en las 
organizaciones incrementa el grado de implicación personal, refuerza el control del trabajador sobre su trabajo, mejora la productividad, aumenta la satisfacción laboral, afecta positivamente al desarrollo de la carrera profesional y, en general, mejora la calidad de la vida laboral de la persona, a la vez que influye positivamente sobre su desarrollo adulto. Por su parte, a nivel grupal y organizacional, la participación mejora la solución de los problemas, aumenta la productividad, la ejecución y eficacia del trabajo global y en grupo, genera un mejor clima organizacional, mejora las actitudes de los individuos, disminuye el absentismo y la rotación, genera una mayor eficiencia organizativa y suele producir un mayor crecimiento económico.

Estos resultados ponen de manifiesto la gran importancia de la participación para el funcionamiento organizacional y el desarrollo de una conducta organizativa más humanizada y satisfactoria, libre de los efectos negativos de los sistemas más autoritarios, que, por otra parte, no por ello son mejores para promover la eficiencia de las organizaciones.

Sin embargo, la voluntad de participar en las decisiones de las empresas que la mayor parte de los trabajadores expresa en los estudios de opinión que se realizan (Quintanilla, 1988: 75), a menudo se ve refutada en el momento en que la democracia se pone en práctica, no sólo por la actitud, más o menos esperada y "comprensible» de los empresarios —en tanto que la participación significa para éstos una cesión de una parte de su poder-, sino también por los propios trabajadores. Así, cuando dicha participación implicaba el desempeño de un papel activo en el desarrollo de ese proceso, había trabajadores que se retraían de ello y, más aún, algunos entendían que implicarse en «las decisiones propias de la dirección» significaba confundir las funciones que les son propias a los trabajadores (Fundación..., 1988: 48).

Una percepción ideológica como la anterior, que concibe la participación como un acto impropio de los trabajadores porque carece de la legitimidad necesaria, además de corresponderse con una visión ideológica cerrada, que valora como más aceptables los costes de renunciar a la posibilidad de decidir, influir o criticar las numerosas cuestiones que pueden afectar a los individuos, que los hipotéticos riesgos de una mayor participación; podría indicar también que algunos trabajadores temen estar incapacitados para desarrollar ciertas funciones distintas a las que habitualmente realizan o que recelan de comprometerse con un mayor número de responsabilidades de las que ya tienen. Todo ello puede comportar que, en determinadas circunstancias, la introducción o el incremento de la participación en la empresa, sobre todo la que comporta una implicación activa en algún órgano decisorio de la empresa, aunque también en otros ámbitos de ésta, podría ser interpretada como algo no deseado y forzado y, en consecuencia, llegar a unos resultados peores que los que se obtenían antes de introducirla o ampliarla.

De hecho, en algunas experiencias en algunos países donde existe una reglamentación de la participación, como por ejemplo Alemania o los países escandinavos, se ha podido constatar la existencia de una cierta distancia entre las estructuras formales de representación de los empleados en los denominados 
"consejos de vigilancia» —organismos de control de la marcha de los acuerdos pactados sobre condiciones de trabajo, incluida la participación- y el resto de trabajadores (Quintanilla, 1988: 95). Así, a estos organismos, a pesar de ostentar legalmente la capacidad de negociar nuevas cotas de poder con la dirección de las empresas, «[...] les resultaba difícil ver la manera de emplear este poder, de forma que, sin apartarse de los objetivos propios de estos consejos [proponiendo un mayor grado de participación, por ejemplo], al mismo tiempo esto repercutiera entre sus representados como algo positivo [...]» (Castillo, 1987: 341), dadas las dudas y, en algunos casos, la apatía con que éstos últimos respondían ante la consecución de más altos niveles en el potencial participativo ya existente.

Por otro lado, como Levinson ya anotó (Levinson, 1977: 87), la mayor parte de las leyes que han regido la participación de los trabajadores en las empresas han sido, en la práctica, bastante restrictivas en cuanto a posibilitar una verdadera participación directa de aquéllos, por lo que los organismos dedicados a la vigilancia participativa, a menudo, se han convertido en meros instrumentos de relaciones sociales que han acabado ocupándose de otras actividades distintas para las que fueron concebidos. Y es que la institucionalización de un derecho como el de la participación en las empresas, cuando no ha sido el resultado de una conquista de los que han de ser sus titulares, puede derivar en una estructura anquilosada y poco eficiente, si los que se benefician objetivamente de ese derecho, no se sienten suficientemente involucrados en su consecución (Hermel, 1990: 136), o bien no acaban de percibir la utilidad que puede tener para mejorar sus condiciones reales de trabajo.

Esto conduce a pensar que las acciones legislativas en favor de la participación suelen ser operativas, sólo en aquellos casos en que previamente se han registrado experiencias participativas con resultado satisfactorio, en tanto que éstas ofrecen un punto de partida para que una posterior intervención legal resulte realmente reforzadora de la participación ya existente (Montero, 1987: 95), sin que esto signifique que la intervención legal no pueda resultar, en ocasiones, muy importante en la difusión de la participación, particularmente en aquellos sectores de actividad o países donde la densidad de pequeñas empresas es elevada o donde la fuerza sindical es significativamente débil. Ambos factores, entre otros, debilitan las condiciones para que la participación pueda irrumpir i generalizarse por sí misma sin ayuda institucional.

En realidad, no es posible analizar los fenómenos de apatía, distanciamiento, autoexclusión u oposición que manifiestan algunos trabajadores ante la participación en la empresa, sin tener presente otros factores que obstaculizan las actitudes proactivas hacia esa participación. Podría suceder que parte del fracaso de algunas experiencias estén relacionadas con los valores de subordinación que numerosos trabajadores han interiorizado profundamente, a veces, durante toda una larga vida laboral, al desarrollar su trabajo en el marco de organizaciones de tipo taylorista, altamente jerarquizadas y fragmentadas en base a las diferencias de poder y del saber formal, por lo que la participación en ámbitos con responsabilidades superiores a las que tienen asignadas por la empresa, 
podría ser considerada como un hecho «no pertinente», al ir en contra del aprendizaje realizado por esos trabajadores dentro de ese marco organizativo, a menudo reforzado por la posesión previa de un ideario actitudinal socializado fuera del ámbito de trabajo - en el mundo familiar, en la comunidad local del individuo o en la propia sociedad a través de los medios de comunicación-, tendente igualmente a la defensa de valores instrumentales, individualistas o, simplemente, fatalistas, ante la presentación de cualquier oportunidad que pueda cambiar una situación de trabajo o de vida, conocida o controlada por el individuo, por muchos beneficios que pueda comportar el hipotético cambio.

En este sentido, la imagen social que algunos trabajadores desarrollan sobre la «sociedad de la clase trabajadora» se corresponde con una visión de tipo «tradicional-deferente» (Poole, 1995: 119). Es decir, bajo el filtro de una imagen de este tipo, numerosos trabajadores, especialmente trabajadores de las pequeñas empresas, aunque también algunos poco cualificados de las grandes empresas, asumen plenamente los valores hegemónicos «propios» de la dirección de la empresa, legitimando así el orden jerárquico establecido por ésta y, por tanto, su posición subordinada dentro de este orden, como consecuencia, entre otras cosas, de la "proximidad" psicofísica que proporcionan las relaciones paternalistas que suelen aparecer en ciertos contextos de trabajo entre empleado y mando o directivo, por lo que se tiende a desdibujar las diferencias sociales objetivamente existentes y, como consecuencia de ello, a valorar las cesiones de poder de la dirección en favor de los trabajadores, como algo incomprensible y hasta negativo.

También, esta vez en un sentido opuesto, no han de descartarse las posiciones reticentes a la participación provenientes de una percepción crítica del fenómeno, en este caso, de aquellos trabajadores que manifiestan —en especial cuando la participación responde a una iniciativa de la propia empresa - un cierto recelo por integrarse en un mayor grado en la gestión de las actividades de la empresa, al entender que participar más de lo requerido por la relación contractual, puede significar la pérdida de más autonomía de la que ya pierden cuando entran en la empresa, en tanto que individuos nominalmente libres, pero también como grupo o hasta como clase social (Castro, 1987: 83), en tanto que participar puede significar «colaboracionismo» $\mathrm{o}$ "hacer el juego» a los intereses de la dirección.

Ante éstos y otros resultados similares, al menos aparentemente, de dudoso deseo participativo de los trabajadores, algunos autores como, por ejemplo, Emery y Thorsrud, del Instituto Tavistock de Londres, llegaron a afirmar que quizás la introducción de la participación convendría iniciarla, sólo si se parte de una situación en la que una gran proporción de los trabajadores se vean, no sólo deseosos, sino también capaces de participar, con lo cual se ponía en cuestión la propia esencia filosófica del derecho a participar de éstos, en tanto que «incapaces».

El tema de la capacidad se ha convertido en una de las principales objeciones que se han venido aduciendo por parte de algunos grupos profesionales, 
empresariales e intelectuales, para oponerse a la participación de los trabajadores en la empresa, bajo el pretexto de que éstos carecen de la cualificación $\mathrm{o}$, a veces, más subliminalmente, de la inteligencia potencial necesaria para gestionar eficazmente las tareas "propias» de la empresa. Las dudas sobre tal capacidad, además de ser tendenciosas, carecen de fundamento, porque la capacidad conceptual y de raciocinio básica puede demostrarse que abunda entre los trabajadores, al menos en la misma medida que en los demás grupos profesionales y sociales, al margen de los niveles de inteligencia que puedan existir (Levinson, 1977: 90).

El hecho de que los que dan órdenes estén jerárquicamente por encima de los que las reciben, no indica que aquéllos posean un nivel de inteligencia o de destrezas superiores, sino distintas. La habilidad en la toma de decisiones de los mandos y directivos se adquiere, fundamentalmente, por la práctica y la experiencia que proporciona el rol desempeñado en razón del cargo que se ostenta, más allá de la inteligencia personal o de las dotes innatas de la persona que ocupa ese cargo.

Todos los puestos de gestión reciben un conjunto amplio de todo tipo de información relacionada con la organización, que tiene poco que ver con la inteligencia y la habilidad de quien ocupa el puesto en cuestión (Levinson, 1977: 91). Sin embargo, la posesión de esta información representa una condición necesaria indispensable para adoptar cualquier tipo de decisión, incluidas las de gestión, por lo que afirmar que los trabajadores carecen de capacidad para gestionar ciertas tareas, cuando no poseen esa información, ni se les ha dado la oportunidad de aprender los conocimientos necesarios para traducirla a decisiones correctas, constituye una afirmación sin sentido.

Además, la adquisición del conocimiento que se deriva de la experiencia acumulada por la práctica directa en el puesto de trabajo, suele ser la base cognoscitiva más importante que tienen los trabajadores para, a partir de ella, iniciar la ascensión a puestos de responsabilidad y, en algunos casos, a los de máximo nivel. Si a ello se añade la posibilidad de acceder a una formación adecuada, no pueden haber motivos para pensar que exista una incapacidad superior de los trabajadores, en relación con otros colectivos, para ejercer eficazmente las tareas de gestión u otras equivalentes que se les puedan encomendar. Hace ya muchos años que los trabajadores, a través de los canales sindicales, participan directamente, o de forma delegada, en numerosos organismos públicos en la planificación y programación de la economía de los países occidentales (Levinson, 1977: 94), además de que son los máximos responsables de numerosas organizaciones económicas que operan sobre una gran gama de bienes y servicios, que obtienen unos resultados, tanto o más positivos, que los que alcanzan las empresas privadas convencionales con las que compiten en el mercado.

Además de las formas de empresa alternativas a las sociedades mercantiles tradicionales, como las cooperativas o los distintos tipos de sociedades laborales, altamente extendidas en todos los países occidentales, en las que el papel de los trabajadores en la propiedad y gestión constituye la característica esen- 
cial de esas organizaciones, existe también una dilatada experiencia empresarial en países como Suecia, Noruega, Holanda, Bélgica, Alemania, Austria, EE. UU., etc., donde los sindicatos gestionan, directamente o en colaboración conjunta con los empresarios, un conjunto importante de empresas dedicadas a una variada tipología de sectores de actividad (desde agencias de viaje hasta constructoras, bancos o editoriales), algunas de las cuales, de gran envergadura y con unos resultados, como ya se ha mencionado, positivos, tanto desde el punto de vista social como económico.

Por no hablar de los numerosos organismos y organizaciones de gestión de la formación, en los que colaboran los trabajadores y representantes sindicales, dedicados a la impartición de programas de formación continuada en innumerables materias - incluidas las de gestión empresarial - a los que asisten centenares de miles de trabajadores en activo, la gestión de los cuales exige poseer una capacidad de organización, tanto técnica como económica, ajustada a los requisitos profesionales que se exigen en cualquiera de las otras empresas igualmente dedicadas a este sector de actividad.

En definitiva, no parece que los motivos de la no participación manifestada por algunos trabajadores puedan atribuirse, principalmente, a motivos de indolencia o desinterés personal y, mucho menos, a una supuesta incapacidad intelectual innata para aprender las habilidades necesarias para tomar el tipo de decisiones adecuadas que implica el ejercicio eficaz de la propiedad y la gestión empresarial.

\section{Los condicionantes de la participación}

Aunque el proceso de democratización industrial, en sus diversas formas, intensidades y repercusiones en que se ha concretado, ha sido el resultado de la conjugación de una compleja y diversificada adaptación de cada organización a las específicas condiciones de su entorno interno y externo y que, por eso mismos, los factores que han intervenido en este proceso han sido muy variables y de difícil determinación, es posible apuntar algunos de los principales condicionantes que han influido en el hecho que la evolución de la participación se haya producido de una manera y no de otra.

Así, por ejemplo, desde la esfera societal, el tipo de prácticas más o menos democráticas que existen en el ámbito político y en las formas de interacción social cotidianas que se producen entre los ciudadanos y el Estado, por un lado, y entre los propios ciudadanos cuando actúan en los diversos subsistemas de la propia sociedad, por el otro, son un fiel reflejo del grado en que la democracia está asentada en la sociedad, como principio que guía la toma de decisiones y la solución de los problemas que se presentan. Conforme ese grado de democracia social se ha ido incrustando en la vida ordinaria de la sociedad civil, más son las razones que presionan para que este tipo de prácticas penetren también en la vida social de las organizaciones y empresas.

Además, el auge de los ideales democráticos como metas universalizadas en las actuales sociedades occidentales, está operando como patrón que regula 
la legitimidad de las actuaciones de cualquier organización y, en general, de cualquier acción en la vida pública social de los miembros de esas sociedades, originándose una progresiva conciencia sobre la «indivisibilidad» de la democracia (Tezanos, 1987: 44), que presiona cada vez más fuerte para que se extiendan los hábitos y las prácticas democráticas propios de la esfera político-social al resto de ámbitos del sistema social, incluido el de las organizaciones económicas.

En la creación de esa conciencia han intervenido diversos factores, pero, sin duda, ha sido fundamental el incremento substancial del nivel de instrucción, tanto de la población en general como, en especial, de la población activa, con lo que la capacidad potencial de los trabajadores para el desempeño de tareas de mayor complejidad y responsabilidad ha elevado objetivamente, ampliándose su capacidad para extender y cambiar las fronteras de su experiencia laboral (Poole, 1995: 121) y, así, poder exigir un nuevo trato más participativo en las decisiones que les conciernen. De forma simultánea, el acceso a la dirección de las empresas de personas, igualmente, con un mayor grado de estudios y de cualificación profesional, ha de hacer posible la formación de una nueva cultura de gestión empresarial, más propensa a ver la participación como un hecho, no sólo necesario, sino también positivo, aunque sólo sea por haber comprobado el rendimiento productivo de su puesta en práctica. Sin duda, en ese cambio de actitud, la agitada situación socioeconómica del entorno de estos últimos años, ha desempeñado un papel crucial.

Desde una óptica económica y organizativa, el progresivo tránsito desde una organización de la producción y del trabajo taylorista — caracterizada por la presencia de un elevado grado de alienación y absentismo, así como por una fuerte segregación intelectual del trabajo y una intensa jerarquización del poder y la división de las tareas, tal como se ha apuntado antes-, hacia una de más flexible, tecnificada y, en cierta medida, más cualificada y menos jerarquizada, pero también más exigente para con las actitudes en favor de una mayor implicación y autorresponsabilidad de los trabajadores, ha propiciado la necesidad de percibir la participación de éstos como algo ineludible, si se desean lograr los objetivos empresariales con ciertas garantías, dados los cambios producidos en la mano de obra y en las condiciones de competitividad del actual mercado.

Por otro lado, otro de los factores socioorganizativos que condicionan el grado que puede conseguir la participación de los trabajadores, está relacionado con el tamaño de las organizaciones. La gran dimensión empresarial, en términos ocupacionales, suele funcionar bajo criterios técnicos y burocráticos, por encima de las prácticas paternalistas y de los lazos de amistad, más propios de las pequeñas empresas, tal como ya se ha visto anteriormente, produciéndose así una mayor objetivación y transparencia de las desiguales relaciones sociales de poder que se asocian a los procesos de producción en aquellas empresas. Con ello, se propicia una determinada interacción social que ayuda a que los trabajadores puedan percibir mejor sus intereses comunes frente a los de sus empleadores. De esta manera, se tiende a facilitar la unidad de acción de aquéllos $y$, por ende, las condiciones adecuadas para lograr una organización y sindi- 
cación más robusta para presionar con más fuerza en favor de la participación (Poole, 1995: 143). Ahora bien, la gradual disminución de que ha sido objeto el tamaño medio de las empresas en estos últimos años, ha de valorarse como un comportamiento que, necesariamente, debe haber influido en la baja de la participación, explicando con ello una parte de la ralentización sufrida por ésta durante la última década.

En tercer lugar, a diferencia de lo que, generalmente, sucede en las situaciones de pleno empleo, los períodos de recesión económica suelen conducir a que los sindicatos pierdan poder de negociación, así como a que los propios trabajadores reformulen el orden de sus expectativas laborales en dirección a resolver problemas más acuciantes como el mantenimiento del empleo y de los salarios y, en consecuencia, a relegar o atenuar los impulsos para una mayor participación (Domínguez, 1979: 79). Ahora bien, también es verdad que, a resultas de las crisis económicas, se crean las condiciones para que las empresas pacten con los trabajadores un mayor grado de cooperación de éstos para con la empresa, como vía para poder salir con más facilidad de la crisis, por lo cual puede surgir también una participación superior a la que existía antes de iniciarse la citada crisis (Tezanos, 1987: 48). Por este motivo, la participación de los trabajadores no puede ser considerada simplemente como un fenómeno de los «buenos tiempos».

Sin embargo, en la práctica, la elección de una senda más o menos «democrática» para salir de la crisis, va a depender, en mucho, de los valores culturales que predominen en cada empresa, más allá de las soluciones específicas con que se puedan afrontar los problemas y de las exigencias que el mercado pueda imponer a cada empresa para resurgir de la crisis. En otras palabras, la salida de la crisis puede dar lugar a una mayor participación de los trabajadores, según sea la percepción que cada empresa posea sobre sus recursos humanos: como principal potencial con el cual contar y participar, ya desde el principio, para resolver las consecuencias de las crisis económicas o, por el contrario, como recurso subsidiario, perfectamente prescindible y sustituible por capital o tecnología y para, en todo caso, reprender su contratación - necesariamente en menor medida - una vez superado el ciclo recesivo.

Detrás de esas distintas orientaciones reposan unas determinadas creencias y valores de signo también distinto sobre la concepción que las empresas tienen sobre sus trabajadores. Así, se les puede percibir como seres inmaduros, poco capaces e irresponsables por "naturaleza», que han de ser substituidos en cuanto sea factible y, por tanto, a quienes no es posible ceder más participación que la estrictamente relacionada con la obtención de una mayor productividad de su trabajo, porque ir más allá, es decir, concederles un mayor grado de poder para decidir sobre otros aspectos, podría poner en peligro el principio de autoridad exclusivo de la dirección. Por el contrario, se puede concebir al trabajador como un ser, esencialmente, capaz e inteligente, con derecho a participar directamente en las decisiones que le afectan en la empresa, en tanto que medio para elevar su autoestima como persona, su responsabilidad como trabajador y su libertad como ser humano. 
Por su parte, desde el punto de vista tecnológico, la tecnología puede comportarse como un condicionante importante del poder que tienen los trabajadores para participar en la toma de decisiones de las empresas. Conforme el entorno ha ido provocando un mayor número de situaciones de cambio constante en las empresas y las tecnologías han ido asumiendo, progresivamente, las tareas productivas de los trabajadores y han ido integrando técnicamente las diversas funciones empresariales, la organización del trabajo en la empresa ha tenido que adoptar, como también ya se ha comentado anteriormente, formas de trabajar, cada vez, más flexibles, pero también más participativas, para propiciar la implantación del trabajo en grupo y la polivalencia de sus miembros, dando pie a que, en términos generales, el desempeño de los puestos de trabajo exija de los trabajadores la posesión de una mayor cualificación.

Debido a estos superiores requisitos del puesto de trabajo, el control directo de la dirección sobre la tarea que realizan los trabajadores resulta, cada vez, más difícil de llevarse a cabo, por lo que el poder discrecional de estos trabajadores para la toma de numerosas decisiones, sobre todo las relacionadas con la organización autónoma de su trabajo y la resolución de problemas, se ha elevado significativamente. Es decir, la tecnología y las nuevas formas de organización del trabajo se pueden convertir en un factor propiciador de una mayor participación de los trabajadores en el control de su trabajo, según sea el grado en que los cambios organizativos y tecnológicos hagan depender a los directivos y empresarios de las cualificaciones que sus trabajadores han de poseer para hacer eficiente el uso de la tecnología o de la nueva forma de organizar el trabajo (Fundación..., 1988: 37), en tanto que condición para lograr los objetivos que dieron lugar a la introducción de esos cambios (Fröhlich, 1990-91: 3-23).

Sin embargo, es necesario aclarar que más allá de que los trabajadores posean una elevada cualificación y puedan decidir sobre su trabajo en mayor grado que los menos cualificados, ni las nuevas formas de organización del trabajo, ni las nuevas tecnologías elevan o reducen, por sí mismas, la cualificación de los trabajadores y, aún menos, que eso conlleve, necesariamente, una participación de los trabajadores en la toma de las decisiones importantes de las empresas. El potencial participativo que poseen ambos instrumentos se desencadenará con mayor o menor fuerza, en función, una vez más, del tipo de cultura organizativa que predomine sobre la participación en cada empresa, zona o país, pero también según sea la orientación más o menos favorecedora de la participación que tengan las políticas tecnológicas promovidas gubernamentalmente, de cuáles sean las actitudes empresariales al respecto y, sobre todo, de cuál sea la capacidad sindical para comprometer a los empresarios (Cornfield, 1995: 27-53), para que ese potencial se active en su máximo grado, mediante una gestión conjunta del cambio organizativo y tecnológico, desde el mismo momento en que se toma la decisión de llevarlos a cabo, hasta que finaliza la evaluación de los resultados obtenidos por su implantación.

Finalmente, desde la esfera institucional, el papel del Estado y de los gobiernos puede llegar a ser trascendental para que la participación de los trabajadores en las empresas exista - estimulando y facilitando los procesos de estructuración 
inicial de ésta en las organizaciones- y, en todo caso, si ya existe, para preservarla y propiciar una mayor profundización de ésta y, en último término, para darle un carácter de derecho normativo. Por otro lado, las políticas gubernamentales en materia de participación que se aplican en las administraciones y en las organizaciones y empresas del sector público, actúan como pautas que orientan e influyen al resto de organizaciones y empresas del sector privado, en la medida que establecen un umbral de expectativas en relación con la participación (Poole, 1995: 54) y se convierten en un punto de referencia para el avance de la participación, en general.

Sin embargo, las formas de participación impulsadas por la legislación no siempre son, una vez más, por sí solas garantía de un desarrollo eficaz de los sistemas de participación, ni de que la participación sea aplicada en cuestiones clave. Por estos motivos, es necesario tener en cuenta la influencia de otros factores que pueden reforzar o, por el contrario, anular el desarrollo de los contenidos previstos por las leyes, hasta el punto que en algunos países donde la participación está poco regulada, los acuerdos informales o la negociación colectiva que se ha llevado a cabo entre las partes involucradas, han logrado mayores resultados en materia de participación que los obtenidos por otros países con legislaciones explícitamente desarrolladas, pero poco abiertas a los cambios y muy rígidas para solventar los problemas específicos que cada empresa, territorio o sector de actividad pueda tener en relación con este tema.

En términos generales, allí donde la tradición del movimiento obrero ha sido y, en cierta manera, sigue siendo importante, pero las estructuras políticas y sociales democráticas han sido relativamente débiles, los logros en la democratización del trabajo alcanzados han sido, principalmente, protagonizados por los sindicatos (Bélgica e Italia, por ejemplo). Por su parte, donde los sindicatos han sido tradicionalmente fuertes $\mathrm{y}$, a su vez, las estructuras también han sido altamente democráticas, los altos logros participativos conseguidos han sido el resultado de una combinación entre presión sindical y legislación gubernamental favorable (por ejemplo, los países escandinavos) (Poole, 1995: 184). En una situación intermedia, se sitúan países como, por ejemplo, Alemania u Holanda, en los que la fuerza sindical no ha sido tan elevada como en el caso anterior, pero la legislación estatal ha asumido, en buena medida, la regulación centralizada de la participación, dando lugar a unos niveles participativos también bastante aceptables. Finalmente, en países como España, Portugal o Grecia, ni los sindicatos han logrado imponer cuotas importantes de participación en las decisiones relevantes de las empresas, ni las estructuras se han caracterizado por una larga tradición democrática, ni los gobiernos han forzado, en general, una legislación claramente comprometida con la participación.

Por su parte, el papel de los sindicatos constituye uno de los núcleos fundamentales para el avance de la democracia industrial, pero no sólo por su rol de principales impulsores de la participación de los trabajadores, sino también porque, en tanto que agentes sociales formalmente organizados e institucionalizados, su actitud para con la democracia participativa en el seno de sus propias organizaciones se convierte en una imagen de referencia para la actua- 
ción de los trabajadores. El principio de democracia como guía del funcionamiento interno en estas organizaciones, constituye un requisito previo esencial para que la democracia industrial pueda configurarse en las empresas (Poole, 1995: 167), y en ello, los dirigentes sindicales tienen la máxima responsabilidad para que eso acabe siendo así. La centralización de la negociación colectiva, la progresiva profesionalización de los dirigentes y la ausencia de debate y participación de los afiliados en las organizaciones locales y de los propios trabajadores electores en las empresas tiende, como en cualquier organización de grandes dimensiones, a burocratizar el proceso de toma de decisiones y a impedir una dinámica organizativa democrática, con unos resultados que, además de defraudar las expectativas de sus afiliados y representados, acaba, a menudo, poniendo en cuestión la propia credibilidad de las intenciones democráticas que los sindicatos reclaman para terceros ${ }^{1}$.

Además, los sindicatos tienen siempre planteado el problema de cómo conjugar los objetivos generales que, como organizaciones sociales, persiguen para el conjunto de los trabajadores, con las distintas y específicas aspiraciones que éstos tienen en relación con la participación. En general, para los trabajadores individuales o los grupos de trabajadores, la participación constituye un objetivo, en la medida en que ésta pueda mejorar el entorno y el contenido de sus puestos de trabajo en una dirección de elevar la calidad laboral, enriqueciendo las tareas o mejorando las cuestiones ergonómicas del puesto (Fundación..., 1988: 34), algo que, normalmente, suele incumbir menos a los colectivos de menor cualificación y a aquellos individuos que conciben su trabajo de forma instrumental, lo cual explicaría, al menos en parte, la indiferencia ante la participación que muestran siempre algunos colectivos laborales.

1. El estudio de R. Alós y otros, La transformación del sindicato, de reciente aparición -y, a su vez, continuador de otro realizado en 1992 - sobre las características personales y laborales de los afiliados de CCOO de Cataluña, es una buena muestra para conocer, entre otros aspectos, las valoraciones subjetivas que dichos afiliados tienen acerca de las prácticas, más o menos participativas y democráticas, que se llevan a cabo en el seno de esa organización, en cuanto a la toma de decisiones en el funcionamiento interno ordinario de la vida sindical en los órganos de representación en las empresas y en las sedes centrales del sindicato. En general, los resultados del estudio apuntan a que, entre otras cosas, y a pesar de haberse constatado ciertos avances respecto al estudio precedente, persisten algunas limitaciones, de orden objetivo, pero también subjetivas, en el acceso de la mayoría de los afiliados a esos órganos y, especialmente, a la cúpula de la organización; algo que, desde algunos ámbitos del sindicato viene a justificarse como necesario para garantizar la "eficiencia» de éste para lograr los fines propuestos. Así, la antigüedad y experiencia en el cargo, el nivel de estudios, la cualificación, el género, la edad, la estabilidad contractual, etc., pero también la disponibilidad de tiempo, el temor a hablar en público, la voluntad de compromiso o la capacidad para gestionar un grupo; son algunas de las principales variables que determinan que, en última instancia, sólo una mínima parte de los afiliados acabe accediendo a los cargos de responsabilidad, sin que los mecanismos de renovación que posibilitan los diversos congresos y elecciones sindicales que, periódicamente, se realizan, hayan modificado substancialmente el perfil de los que resultan elegidos para esos cargos. 
En cambio, para los sindicatos, el objetivo fundamental de la participación suele ir más allá de las motivaciones psicológicas y materiales que cada individuo pueda tener ante las condiciones específicas de su trabajo, centrándose en cómo mejorar los resultados en las negociaciones de los convenios colectivos y cómo enfocar sus estrategias ante las políticas que siguen los empresarios para mantener el control de las empresas o, contrariamente, para compartirlo con los trabajadores, por lo que la única solución que tienen los sindicatos para compaginar ambas perspectivas supone aumentar el contacto directo con las necesidades y aspiraciones que los trabajadores tienen en la vida diaria en su puesto de trabajo y, al mismo tiempo, tener que reconocer que la participación de los trabajadores en la empresa no es homogénea, sino que posee distintas intensidades, porque los intereses, al menos subjetivos, de éstos, son también distintos.

\section{Algunas conclusiones}

El avance hacia la democratización de las organizaciones y de las empresas se ha convertido en un fenómeno social irreversible en los países industrializados, porque, a pesar de la gran diversidad de grados y formas que ha adquirido la práctica participativa, tanto dentro de cada país como entre los propios países, constituye un objetivo histórico del movimiento sindical de estos países, además de que las experiencias que se han llevado a cabo muestran, en general, unos resultados favorables para la mejora de las condiciones psicosociológicas y materiales de los trabajadores, pero también porque esas experiencias demuestran que son útiles para cubrir los retos que plantea el nuevo entorno competitivo a los empresarios, en tanto que la implicación y participación de los trabajadores se ha convertido en un factor estratégicos para aquéllos, haciendo que, cada vez, sea más impensable solicitar la adhesión y cooperación de los trabajadores para con la empresa, si éstos no son partícipes de las decisiones que se toman en ésta.

En realidad, la importancia de la participación no reside tanto en los resultados materiales que se alcanzan, ni en el tipo de instituciones o estructuras que se emplean para lograr su implantación y desarrollo, sino en los efectos más intangibles, directos e indirectos, que la participación provoca, sobre todo a largo plazo, en el cambio de comportamientos y actitudes de las partes involucradas, como consecuencia del conocimiento de los objetivos e intenciones que tiene la otra parte y que proporciona, justamente, la negociación continuada para consensuar acuerdos durables y aceptables por ambas partes.

Por otro lado, la participación en las organizaciones, para que sea realmente efectiva, requiere de la integración activa de todos los individuos, y no sólo de los que son objeto de delegación para vigilar su cumplimiento. Sin estos requisitos, no hay verdadera participación; se producen, en todo caso, manifestaciones cercanas, pero nunca formas de participación rigurosamente aceptables (Quintanilla, 1988: 111). En este sentido, la «dirección participativa» o modelo que propugna la participación de los trabajadores top down, «desde arriba 
hacia abajo", no constituye una verdadera participación, sino, como dice Quintanilla citando a Crozier (Croizer, 1970: 221), es un «regalo» de la dirección que, aunque puede resultar eficaz para elevar la producción, puede representar un "engaño" para los trabajadores, si no se ve complementada con una participación de tipo botton up, «de abajo hacia arriba».

De hecho, la confluencia de ambas perspectivas parece que responde a la filosofía que guía a los modelos de participación más recientes, al menos eso es lo que suele suceder con el modelo institucionalizado de «co-determinación» alemán y, más específicamente, con el de "democracia industrial» instituido en Suecia, ambos legalmente respaldados desde los años setenta, que han dado buenos resultados y han marcado una línea de desarrollo, repetidamente imitada por otras tentativas de democratización, a la vez que se han constituido en un referente ineludible para el análisis de la participación en la empresa.

De otra parte, a pesar de que algunas de las experiencias desarrolladas no han dado todos los resultados esperados o, simplemente, han fracasado en su intento por motivos muy diversos, algunos aquí ya comentados, el objetivo de democratizar la empresa como parte de la sociedad democrática debe seguir siendo defendido, especialmente, por los sindicatos (Tezanos, 1987: 10). Y esto debe hacerse por muchas razones, pero sobre todo porque es necesario mantener la congruencia ética y política de alcanzar una meta acorde con las aspiraciones históricas del movimiento obrero y de la propia sociedad más liberal y progresista, ya que continúa siendo necesario obtener parcelas de poder en la empresa que permitan defender mejor los intereses de los trabajadores, sobre todo, si esto se consigue de forma concertada con la patronal y no sólo por la vía institucional, para así eludir posibles retrocesos por cambios en el poder político desfavorables a la participación y, finalmente, porque constituye una táctica para no quedar al margen de la iniciativa participativa que puedan propugnar los mismos empresarios y evitar, con ello, una potencial dualidad quebrantadora entre trabajadores que entren en la lógica participativa empresarial y trabajadores que no la acepten.

La democracia industrial hace tiempo que ha dejado de ser un fenómeno utópico o aislado en unas pocas empresas y en unos pocos países. Hoy existen, más que nunca, las necesarias condiciones de nivel de vida material e intelectual, de tecnologías y formas organizativas con potencial altamente participativo y de la suficiente oferta formativa para la capacitación de los trabajadores y de los propios empresarios, como para que se puedan ejercer los máximos niveles de participación en la propiedad y en la gestión de la empresa, tanto por propia iniciativa de las empresas, como, sobre todo, por iniciativa conjunta. Si esto no ocurre, será porque otros condicionantes de tipo ideológico o político-institucionales siguen oponiéndose a los objetivos de democratizar y humanizar algo tan humano como el trabajo humano, o porque, quien históricamente ha sido su agente más propulsor, el movimiento sindical, carece hoy del poder que tuvo en otros momentos para forzar al resto de agentes implicados, nuevos avances en esa dirección. 


\section{Bibliografía}

Alós, Ramon y otros (2000). La transformación del sindicato: estudio de la afiliación de CCOO de Cataluña. Barcelona: Viena Serveis Editorials.

BEIRNE, M.; RAMSAY, H. (1992). Information technology and workplace democracy. Londres: Routledge.

CASTILlO, José (1986). «La democracia industrial: paradojas del poder en las organizaciones formales». Sistema, 73. Madrid.

COMISIÓN EUROPEA (1997). Libro verde sobre cooperación para una nueva organización del trabajo. Bruselas.

CORNFIElD, Daniel B. (1995). "Potencial participativo de las nuevas tecnologías y sindicalismo». Sociología del Trabajo, 23, invierno. Madrid.

Crozier, Michel (1984). No se cambia la sociedad por decreto. Alcalá de Henares: Instituto Nacional de Administración Pública.

Fundación Europea Para la Mejora de las Condiciones de Vida y de Trabajo (1988). Análisis de la participación: balance de los estudios en materia de participación de los trabajadores en las decisiones de la empresa. Luxemburgo: Office des Publications Officielles des Communautés Européennes.

- (1998). Nuevas formas de organización del trabajo; ¿Puede Europa aprovechar su potencial? Luxemburgo: Office des Publications Officielles des Communautés Européennes.

GARCÍA-NiETO, Juan N. (1978). La participación de los trabajadores en la gestión empresarial en España. Ginebra: Instituto Internacional de Estudios Laborales.

GiMÉNEZ, José Luís (1995). «La participación de los trabajadores en la empresa». En GiMÉNEZ, José Luis y otros. Lectures de Sociologia de l'Empresa. Barcelona: Rey.

Gladstone, Alan; Ozaki, Muneto (1994). Participación de los trabajadores en las empresas, nuevas tecnologías y programas de formación. Ministerio de Trabajo y Seguridad Social.

GORZ, Andrè (1977). Crítica de la división del trabajo. Barcelona: Laia.

- (1986). Los caminos del paraiso. Para comprender la crisis y salir de ella. Barcelona: Laia.

HeCKSCHER, Ch. (1993). El nuevo sindicalismo. La participación del trabajador en la empresa en transformación. Madrid: Ministerio de Trabajo y Seguridad Social.

LEVINSON, Charles (1977). La democracia industrial. Madrid: Asociación para el Progreso de la Dirección.

LuCAS, Antonio (1984). «Perspectivas sociológicas para el estudio de la democracia industrial». Revista Internacional de Sociología, 49, enero-marzo. Córdoba.

MARTín ARTILES, Antonio (1999). «Organización del trabajo y nuevas formas de gestión laboral». En Miguélez, F.; PRIETO, C. Las relaciones de empleo en España. Madrid: Siglo XXI.

MARZAL, Antonio (1976). Empresa y democracia económica. Madrid: Guadiana.

MCGregor, D. (1969). La participación en la industria. Barcelona: Hispano-Europea. OIT (1981). Participación de los trabajadores en las decisiones de la empresa. Ginebra. Poole, Michael (1995). Hacia una nueva democracia industrial. Madrid: Ministerio de Trabajo y Seguridad Social.

REBOLLO, Oscar y otros (1993). El sindicalismo a través de sus protagonistas. Barcelona: CERES.

SARACÍBAR, José Antón (1987). «El reto sindical de la participación obrera en España». En Tezanos, J.F. La democratización del trabajo. Madrid: Sistema. 
TeZANOS, José Félix (1987). La democratización del trabajo. Madrid: Sistema.

VILLA, Luís Enrique (1980). La participación de los trabajadores en la empresa. Madrid: Instituto de Estudios Económicos.

WICKHAM, James (1998). «Squaring the circle? Participation, innovation and employment». European Review of Labour and Reseach, vol. 4, núm. 2. Bruselas. 